\title{
Aceleração da Classificação de Lavouras de Milho com MPI e Estratégias de Paralelismo
}

\author{
Anthony Vanzan ${ }^{1}$, Gabriel Rustick Fim ${ }^{1}$, Greice Aline Welter ${ }^{1}$, Dalvan Griebler ${ }^{1,2}$ \\ ${ }^{1}$ Laboratório de Pesquisas Avançadas para Computação em Nuvem (LARCC), \\ Faculdade Três de Maio (SETREM), Três de Maio, Brasil \\ ${ }^{2}$ Escola Politécnica, Grupo de Modelagem de Aplicações Paralelas (GMAP), \\ Pontifícia Universidade Católica do Rio Grande do Sul (PUCRS), Porto Alegre, Brasil
}

\{vanzan.anthony,gabifimtm, greicewelter8, dalvangriebler\}@gmail.com

\begin{abstract}
Resumo. Este trabalho visou acelerar a execução de um algoritmo de classificação de lavouras em imagens áreas. Para isso, foram implementadas diferentes versões paralelas usando a biblioteca MPI na linguagem Python. A avaliação foi conduzida em dois ambientes computacionais. Conclui-se que é possível reduzir o tempo de execução a medida que mais recursos paralelos são usados e a estratégia de distribuição de trabalho dinâmica é mais eficiente.
\end{abstract}

\section{Introdução}

A implementação de novas tecnologias no agronegócio, que aprimorem as técnicas já desenvolvidas ou até mesmo criem novas formas de realizar tarefas, otimizando o tempo e possibilitando aferir os dados com maior precisão, são de suma importância para o desenvolvimento da área. Cada dia mais os avanços tecnológicos permitem a implementação de tecnologias de inteligência artificial (IA) juntamente com imagens aéreas providas por veículos aéreos não tripulados (VANTs). A análise destas imagens a partir de IA requer alto poder computacional e a aplicação de técnicas de computação paralela para obter os resultados em tempo hábil.

Um programa para classificação de lavouras de milho em linguagem Python, chamado de classificador [Vanzan et al. 2020], foi desenvolvido no escopo do projeto AgroComputação ${ }^{1}$, onde o objetivo é desenvolver soluções que auxiliam na medição da densidade da plantação através da análise de imagens capturadas a partir de VANTs e aplicando algoritmos de aprendizado profundo (Deep Learning). Neste artigo, o foco é implementar e avaliar diferentes versões paralelas do classificador, com o objetivo de acelerar o processo de classificação de áreas de lavouras, especificamente na cultura do milho. Além de contribuir com a aceleração deste processo, o artigo visa avaliar diferentes padrões paralelos, estratégias de distribuição de trabalho e ambientes computacionais.

$\mathrm{Na}$ literatura, a paralelização de programas escritos em Python tem sido realizada usando a biblioteca MPI, já bastante utilizada e consolidada nas linguagens $\mathrm{C} / \mathrm{C}++$ e Fortran. Nota-se que os estudos que avaliam o uso para processamento paralelo apresentaram desempenho muito similar entre as linguagens [Dalcin et al. 2011]. Mais especificamente, no contexto da paralelização de aplicações de aprendizado profundo, o foco tem sido a parte do treinamento [Ma et al. 2017]. No caso deste trabalho, o classificador é um programa que usa um modelo já treinado. Este artigo está estruturado da seguinte forma. Na seção 2 é descrito o classificador e como ele foi paralelizado. Na seção 3 são discutidos os resultados obtidos em dois ambientes computacionais.

\footnotetext{
${ }^{1}$ ACST - Projeto AgroComputação com apoio da SETREM e TECNICON: http://acst.setrem.com.br/
} 


\section{Paralelização do Classificador}

A Figura 1(a) ilustra as etapas do programa classificador em sua versão sequencial. Ele começa realizando o recorte de uma ortofoto em imagem de $32 \times 32$ Pixeis. Esse é o tamanho da entrada suportado pela LeNet5 [Y. Lecun and Haffner 1998], sendo esta a arquitetura de rede neural utilizada pelo classificador. Essas imagens recortadas e as coordenadas de onde estas foram retiradas são anexadas a uma lista como uma tupla. Quando toda a ortofoto tiver sido recortada, o algoritmo realizará a classificação das imagens. Após ter transformado o recorte em grayscale, é chamado um modelo de rede neural LeNet5 prétreinado para classificar em 'Milho' ou 'Não Milho', caso o recorte seja classificado em 'Não Milho' o mesmo é devolvido em grayscale, estas imagens são adicionadas novamente a uma lista para posterior remontagem da ortofoto uma vez que todas as imagens foram processadas, como é mostrado na Figura 1.

Para realizar a paralelização foram utilizados dois padrões paralelos, um utilizando a estratégia em lotes e outro em stream, usando a arquitetura Master/Worker e Farm respectivimente. Para cada um dos padrões, duas versões foram criadas, uma implementando a distribuição de trabalho round-robin e outro implementando a distribuição de trabalho on-demand. Essas opções são usadas recorrentemente na literatura e obtiveram bom desempenho em outros estudos [McCool et al. 2012, Andrade et al. 2014].

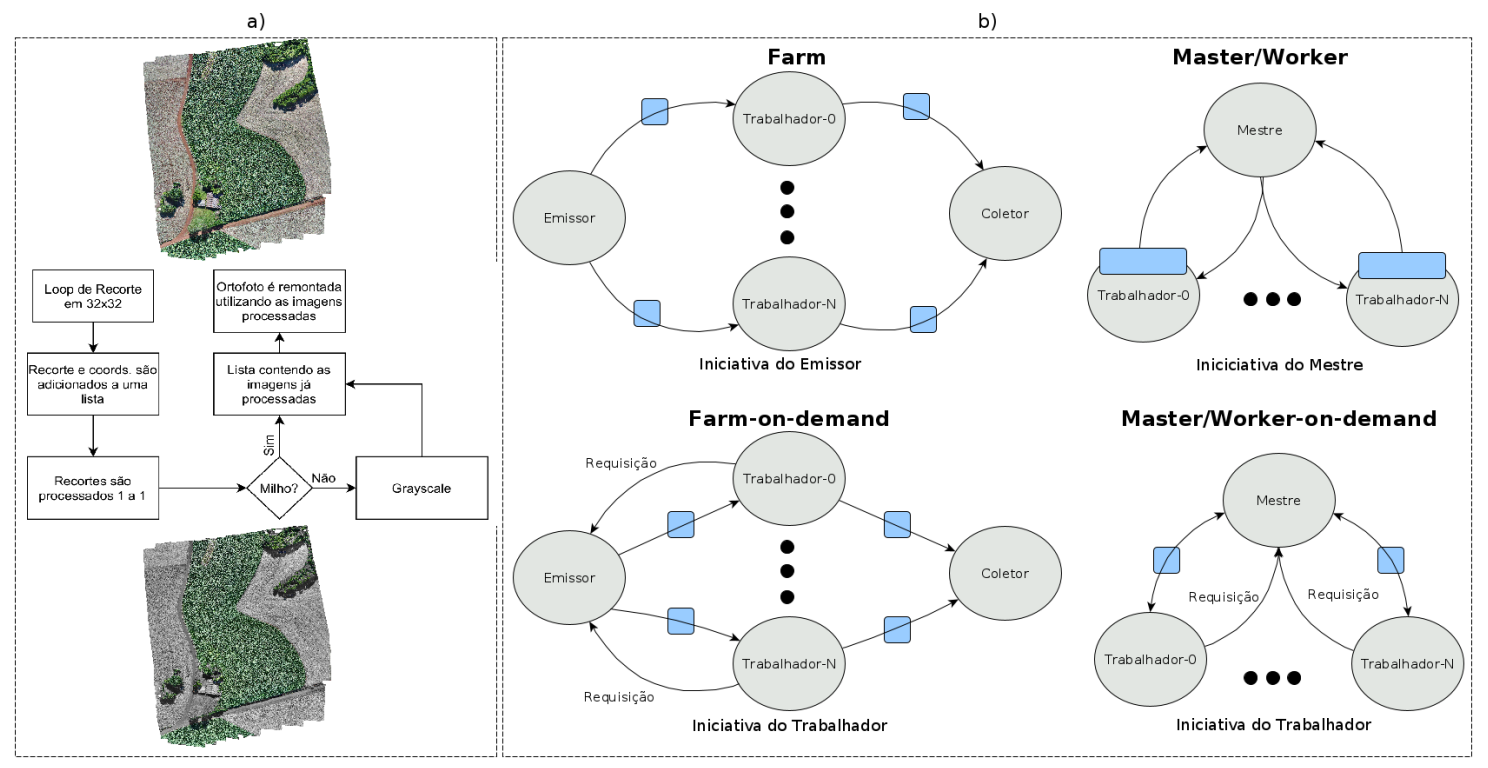

Figura 1. Representação gráfica das versões do classificador.

Na Figura 1(b), as versões Master/Worker utilizaram o mesmo tipo de fila implementado no algoritmo Serial. Na distribuição de trabalho round-robin, o Mestre realiza a divisão e envio dos recortes da ortofoto para os Trabalhadores realizarem o processamento e devolverem estes ao Mestre para montagem da ortofoto processada. Já no Farm, a lista não se faz necessária pois o Emissor realiza o recorte de 32x32 pixeis da ortofoto e o envia imediatamente a um Trabalhador, que uma vez concluído o processamento, a envia ao Coletor para montagem da ortofoto processada. Nas distribuições de trabalho on-demand, a iniciativa parte dos Trabalhadores. Estes requisitam ao Mestre ou Emissor um trabalho quando ociosos. Todas as versões foram paralelizadas com a biblioteca OpenMPI em Python3 para a troca de mensagens, mais especificamente através de comunicação bloqueante. 


\section{Resultados}

Os experimentos foram executados na infraestrutura do LARCC ${ }^{2} \mathrm{em}$ dois ambientes computacionais. O primeiro ambiente, apelidado de StormBreaker, possui como sistema operacional o Ubuntu Server 20.04.1 LTS (Focal Fossa) (Kernel 5.4.0-62) e softwares Python 3.7.7, NumPy 1.19.2, OpenCV 3.4.2, PyTorch 1.7.1, mpi4py 3.0.3 e OpenMPI 4.0.3. Seu hardware é composto por um processador Intel i9-7900x (20 threads), 32GB de RAM, discos de armazenamento 4x 2TB Sata III 6.0GB/s em raid 5.

O segundo ambiente apelidado de Nebula-Cloud é construído de duas máquinas HP Proliant DL385 G6, onde cada uma delas possuí um processador AMD Opteron $24252100 \mathrm{MHz}$ Six-Core (totalizando 24 threads), 32 GB de memória RAM DDR3 e 8 discos de 146 GB de armazenamento em RAID 5. Cada máquina possui 5 interfaces de rede Gigabit. A Nuvem configurada utiliza-se da ferramenta OpenNebula 5.12.0.3 com o virtualizador KVM 5.4.0-1031. Para o cenário de testes, foram criados 6 máquinas virtuais de mesma configuração (4 vCPUS, 6GB de memória RAM e 20GB de disco), as quais foram configuradas como um cluster Beowulf com NFS para compartilhamento de arquivos. Essa configuração do ambiente de nuvem visou evitar o uso de hyper-threading, uma vez que foi observado nenhum benefício ao executar no ambiente da Stormbreaker (Figura 2). As máquinas virtuais, onde as versões do classificador foram testadas, possuem as mesmas versões de software: Sistema operacional Ubuntu Server 20.04.1 LTS (Focal Fossa) (5.10.10-051010-generic) com Python 3.8.5, NumPy 1.19.5, OpenCV 4.2.0, PyTorch 1.7.1, mpi4py 3.0.3 e OpenMPI 4.0.3 instalados.
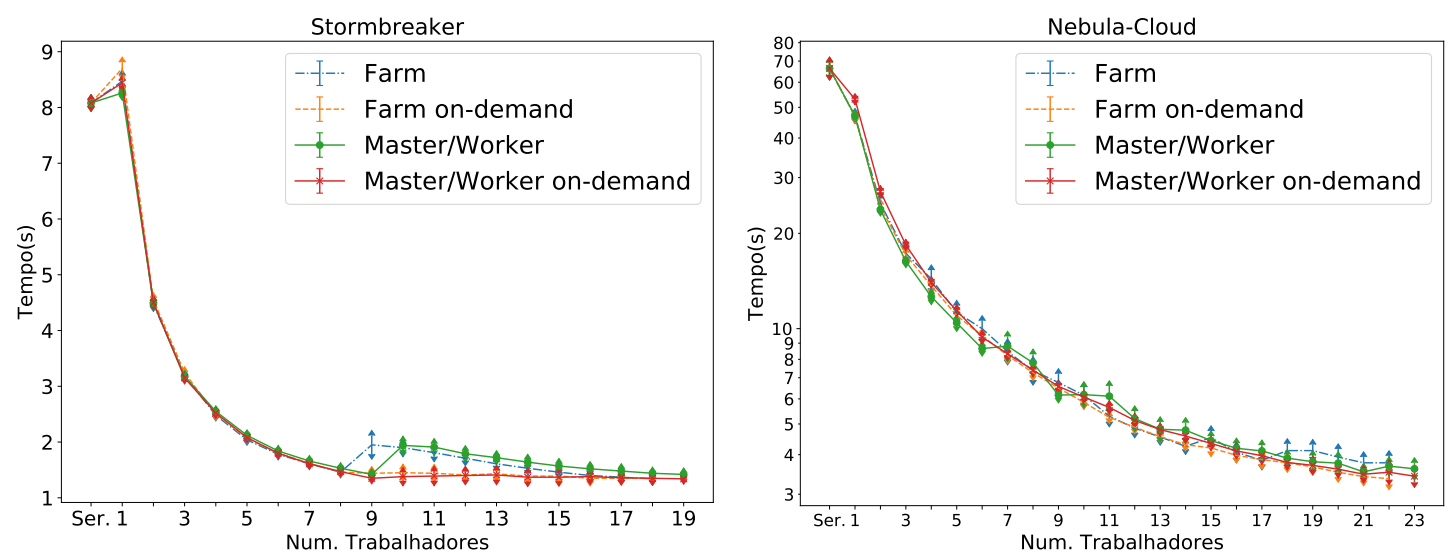

Figura 2. Resultados dos testes nos ambientes.

Para avaliação das versões paralelas, foi utilizada uma ortofoto de $3417 \times 4095$ pixeis, que consequentemente produz 13664 imagens de 32x32 pixeis a serem classificados e remontados pelo classificador. A ortofoto apresenta um exemplo real de uma imagem que seria classificada pelo algoritmo, englobando um ou mais milharais. Na Figura 2 são apresentados os gráficos dos testes, repetindo intercaladamente 30 vezes cada configuração de trabalhadores. Os gráficos apresentam o tempo de execução em segundos (eixo y) em relação a quantidade de trabalhadores (eixo x). Cada linha do gráfico é uma versão paralela diferente do classificador. Junto dos pontos nas linhas, são plotados o desvio padrão das 30 execuções, onde para a maioria das configurações é quase imperceptível. É possível observar que ambos os padrões implementados usando a estratégia de distribuição de trabalho on-demand se sobressaíram. Com isso, é possível concluir que a distribuição

\footnotetext{
${ }^{2}$ Laboratório de Pesquisas Avançadas para Computação em Nuvem: http://larcc.setrem.com.br/
} 
de carga tem impacto no desempenho. Nota-se também que na Stormbreaker, que possui um processador mais novo, o tempo de execução da versão sequencial é muito menor se comparado ao cluster montado na nuvem privada Nebula-Cloud, conforme era esperado. Ainda na Stormbreaker, o tempo de execução das versões paralelas não diminui mais quando entra na zona de uso do hyper-threading para as versões on-demand. Ao invés disso, nota-se que versões round-robin apresentaram um aumento no tempo de execução com 9 trabalhadores no padrão F arm e 10 trabalhadores no padrão Master/Worker. Isso é porque o padrão Farm possui também dois processos concorrentes (Emissor e Coletor) enquanto que o Master/Worker possui apenas um (o Mestre). Além disso, é visível que esta concorrência por recursos gera um desbalanceamento de carga entre os trabalhadores. Embora a estratégia on-demand demande um número maior de mensagens, ela acaba sendo mais eficaz neste aspecto.

No ambiente Nebula-Cloud, plotado em escala logarítmica, observa-se que a versão Master/Worker obteve uma leve superioridade nos resultado até 6 Trabalhadores e após isso, oscilou bastante e tornou-se menos eficiente que as demais versões. Notase também uma oscilação para a versão Farm, atingindo seus melhores resultado entre 11 e 14 trabalhadores. Concluí-se que este comportamento em ambas as versões é devido ao impacto do balanceamento de carga, uma vez nas versões on-demand, o comportamento se mostrou estável. Farm on-demand foi a versão que melhor se saiu a partir de 10 Trabalhadores. Por fim, nota-se que o tempo de execução foi diminuindo quase que logaritmicamente, pois não foi usada propositalmente a tecnologia hyper-threading.

\section{Conclusão}

Concluiu-se que é possível reduzir o tempo de execução a medida que mais recursos paralelos são usados e que a estratégia de distribuição de trabalho on-demand seria a mais eficiente para o objetivo que os algoritmos buscam alcançar. Como estudo futuro, planeja-se realizar testes comparativos com outros padrões paralelos e a aplicação de métricas como SpeedUp dentro dos algoritmos desenvolvidos, visando diminuir ainda mais o tempo necessário para o processamento e classificação das áreas em uma imagem.

Agradecimentos Os autores agradecem ao Laboratório de Pesquisa Avançada em Computação em Nuvem (LARCC/SETREM, Brasil) por fornecer recursos de computação.

\section{Referências}

Andrade, H. C., Gedik, B., and Turaga, D. S. (2014). Fundamentals of stream processing: application design, systems, and analytics. Cambridge University Press.

Dalcin, L. D., Paz, R. R., Kler, P. A., and Cosimo, A. (2011). Parallel distributed computing using Python. Advances in Water Resources, 34(9):1124 - 1139.

Ma, H., Mao, F., and Taylor, G. W. (2017). Theano-MPI: A Theano-Based Distributed Training Framework. In Euro-Par 2016: Parallel Processing Workshops, pages 800813, Cham. Springer.

McCool, M., Reinders, J., and Robison, A. (2012). Structured parallel programming: patterns for efficient computation. Elsevier.

Vanzan, A., Fim, G. R., Welter, G. A., Sausen, M. C., and Griebler, D. (2020). Algoritmo de Deep Learning para Classificação de Áreas de Lavaoura com VANTs. In 22 Salão de Pesquisa Setrem (SAPS), page 5, Três de Maio, RS, Brazil. Sociedade Educacional Três de Maio.

Y. Lecun, L. Bottou, Y. B. and Haffner, P. (1998). Gradient-based learning applied to document recognition. Proceedings of the IEEE, vol. 86, no. 11, pp. 2278-2324. 